\title{
Uncertainty Assessment of the SeaWiFS On-Orbit Calibration
}

\author{
Robert E. Eplee, Jr. ${ }^{a}$ Gerhard Meister, ${ }^{b}$ Frederick S. Patt, ${ }^{a}$ \\ Bryan A. Franz, ${ }^{b}$ and Charles R. McClain ${ }^{b}$ \\ ${ }^{a}$ Science Applications International Corporation, Beltsville, Maryland 20705, USA \\ ${ }^{b}$ NASA Goddard Space Flight Center, Greenbelt, Maryland 20771, USA
}

\begin{abstract}
Ocean color climate data records require water-leaving radiances with $5 \%$ absolute and $1 \%$ relative accuracies as input. Because of the amplification of any sensor calibration errors by the atmospheric correction, the $1 \%$ relative accuracy requirement translates into a $0.1 \%$ long-term radiometric stability requirement for top-of-theatmosphere radiances. The rigorous on-orbit calibration program developed and implemented for SeaWiFS by the NASA Ocean Biology Processing Group (OBPG) Calibration and Validation Team (CVT) has allowed the CVT to maintain the stability of the radiometric calibration of SeaWiFS at $0.13 \%$ or better over the mission. The uncertainties in the resulting calibrated top-of-the-atmosphere (TOA) radiances can be addressed in terms of accuracy (biases in the measurements), precision (scatter in the measurements), and stability (repeatability of the measurements). The calibration biases of lunar observations relative to the USGS RObotic Lunar Observatory (ROLO) photometric model of the Moon are 2-3\%. The biases from the vicarious calibration against the Marine Optical Buoy (MOBY) are 1-2\%. The precision of the calibration derived from the solar calibration signal-tonoise ratios are $0.16 \%$, from the lunar residuals are $0.13 \%$, and from the vicarious gains are $0.10 \%$. The long-term stability of the TOA radiances, derived from the lunar time series, is $0.13 \%$. The stability of the vicariouslycalibrated TOA radiances, incorporating the uncertainties in the MOBY measurements and the atmospheric correction, is $0.30 \%$. These results allow the OBPG to produce climate data records from the SeaWiFS ocean color data.
\end{abstract}

Keywords: SeaWiFS, lunar calibration, vicarious calibration, radiometric stability, radiometric uncertainty

\section{INTRODUCTION}

One goal of climate change research is to discern small secular trends in geophysical processes that may have comparatively large daily, seasonal, annual, or longer-scale periodic signals. This research requires remote sensing data from instruments with long-term radiometric stability, where the radiometric uncertainty in the data is less than the magnitude of the possible climate change signal. For ocean color data, the radiometric requirements are $5 \%$ absolute and $1 \%$ relative accuracies on the water-leaving radiances. ${ }^{1}$ Because open-ocean reflectances are low, approximately $90 \%$ of the top-of-the-atmosphere (TOA) signal observed by ocean color satellite instruments is due to scattering of sunlight by gases and aerosols within the atmosphere. The ocean color atmospheric correction algorithm must remove this signal to yield the water-leaving radiances. Uncertainties in the sensor calibration and in the atmospheric correction algorithm require a vicarious calibration of the sensor/atmospheric correction algorithm system that is independent of time to achieve these accuracy requirements. ${ }^{2}$ Because of the amplification of any errors in the sensor calibration by the atmospheric correction process, the $1 \%$ relative accuracy requirement on water-leaving radiances translates into a $0.1 \%$ long-term radiometric stability requirement for TOA radiances. ${ }^{3}$

One requirement for making full use of the SeaWiFS ocean color data is a quantification of the uncertainties in the calibrated top-of-the-atmosphere (TOA) radiances, particularly for Earth observations. The on-orbit calibration data from which these uncertainties can be characterized include lunar observations, solar diffuser

Further author information:

R.E.E.: Robert.E.Eplee@nasa.gov, 3012860953 
observations, gain calibrations, and vicarious calibrations. In this paper, the NASA Ocean Biology Processing Group (OBPG) Calibration and Validation Team (CVT) will address the uncertainties in the calibrated TOA radiances in terms of accuracy (biases in the measurements), stability (repeatability of the measurements over time), and precision (scatter in the measurements). The mean lunar residuals from the USGS RObotic Lunar Observatory (ROLO) photometric model of the $\mathrm{Moon}^{4-6}$ and the vicarious gains derived from the Marine Optical BuoY (MOBY) $)^{7,8}$ provide information on the accuracy of the radiances. The scatter in the lunar residuals, the signal-to-noise ratios determined on orbit from the solar diffuser measurements, and the uncertainties in the vicarious gains provide information on the precision of the radiances. The limits on the residual time drift in the calibrated lunar observations provide information on the long-term stability of the radiances.

The fully-calibrated lunar time series, with the long-term radiometric corrections applied, is the primary data set for this uncertainty analysis. The oversampling corrections (primarily) and geometry corrections (secondarily) required for the lunar data analysis give rise to systematic errors in the calibrated data that must be accounted for in the assessment of the uncertainties for TOA Earth radiances. In addition, the drift in the 3:1 Gain ratio for Band 7 requires a correction of the Band 7 Earth data which must be taken into account as part of the long-term stability analysis.

\section{CALIBRATION OF ON-ORBIT DATA}

For any SeaWiFS observation the total signal is defined as the dark-subtracted counts of the observation, converted to radiance, then corrected for focal plane temperature, response-versus-scan angle (RVS), and mirror-side reflectance effects: ${ }^{9,10}$

$$
L_{T}(\lambda, t)=\left(C_{o}(\lambda, t)-C_{d}(\lambda, t)\right) K_{c}(\lambda)\left[1+K_{T}(\lambda)\left(T(\lambda, t)-T_{r e f}(\lambda)\right)\right] K_{r v s}(\lambda, p x l) K_{m s}(\lambda, i, t)
$$

where:
$\lambda \equiv$ SeaWiFS band
$t \equiv$ time of the observation
$p x l \equiv$ pixel number in the along-scan direction
$i \equiv$ Mirror Side 1 or Mirror Side 2
$C_{o} \equiv$ output counts for the observation
$C_{d} \equiv$ dark counts for the observation
$K_{c} \equiv$ counts-to-radiance conversion coefficients
$K_{T} \equiv$ focal plane temperature correction factors
$T \equiv$ focal plane temperature for the observation
$T_{\text {ref }} \equiv$ focal plane reference temperature $=16 \mathrm{C}$
$K_{\text {rvs }} \equiv$ RVS correction factors
$K_{m s} \equiv$ mirror-side reflectance correction factors.

A thorough discussion of SeaWiFS instrument calibration will take place in Eplee et al.(2012), ${ }^{11}$ so a summary of the uncertainties in the calibration coefficients determined from the prelaunch instrument characterization $\left(K_{c}\right.$, $\left.K_{r v s}\right)$ and from the detailed on-orbit calibration data analysis $\left(K_{T}, K_{m s}\right)$ are presented in Table 1. Uncertainty in counts-to-radiance conversion coefficients $K_{c}$ is the absolute calibration uncertainty and the primary source of the instrument calibration bias. Uncertainty in the RVS correction $K_{r v s}$ is only significant at the end of scan and is smaller than uncertainties in the atmospheric correction (of Earth data) at large optical paths. Uncertainty in focal plane temperature correction $K_{T}$ is minimized during the lunar data analysis and contributes to the precision estimates. Uncertainty in the mirror-side correction $K_{m s}$ is much smaller than a single instrument count and is essentially negligible. This paper will examine the on-orbit instrument calibration and the corrections computed on orbit for application to the TOA radiances $L_{T}$. 
Table 1. Instrument Calibration Uncertainties. ${ }^{*}$ Bands $1-7 .{ }^{+}$Band 8.

\begin{tabular}{|c|c|c|c|c|}
\hline \multicolumn{2}{|l|}{ Coefficient } & Range & Uncertainty & Applicability \\
\hline Counts to Radiance & $K_{c}$ & & $4 \%^{10}$ & Calibration Bias \\
\hline Response vs Scan Angle & $K_{\text {rvs }}$ & $0.5-1.5 \%$ & $0.3 \%^{9}$ & End of Scan \\
\hline Focal Plane Temperature & $K_{T}$ & $\begin{array}{l}0.04-0.09 \%^{*} \\
0.16-0.27 \%^{+}\end{array}$ & $0.02 \%^{11}$ & Precision \\
\hline Mirror Side & $K_{m s}$ & $0.1 \%$ & $0.01 \%^{11}$ & Precision \\
\hline
\end{tabular}

\subsection{Lunar Data}

The lunar time series (with viewing geometry corrections, oversampling corrections, and coherent noise corrections applied) are shown in Fig. 1. Two different decay mechanisms are responsible for changes in the radiometric response for the bands. The first mechanism caused a rapid change in response that decayed away after the initial couple of years of the mission; there is no current candidate for this short-term mechanism. The second mechanism has been in effect over the entire mission; there are two different long-term response degradation processes occurring for SeaWiFS. The shorter wavelength bands (Bands 1-4) show a degradation that decreases with increasing wavelength and is most likely caused by yellowing of the instrument optics on orbit. The longer wavelength bands (Bands 5-8) show a degradation that increases with increasing wavelength and is most likely caused by charged-particle induced damage to the silicon photodiodes. As the plots show, the degradation over the mission is $\sim 1 \%$ for Bands $3-5$, is $\sim 3 \%$ for Bands 1,2 , and 6 , is $\sim 9 \%$ for Band 7 and is $\sim 21 \%$ for Band 8. With the short-term and long-term decay mechanisms at work, the optimum fitting functions for the time series are either simultaneous exponentials in time (with short-period and long-period time constants) or are single exponentials (with short period time constants) plus linear functions of time. The fits to these time series track the long-term radiometric instrument response for each band, so the inverses of the fits are the long-term radiometric corrections $K_{r c}$ :

$$
\begin{aligned}
& \frac{1}{K_{r c}\left(\lambda_{1,2}, t\right)}=A_{0}(\lambda)-A_{1}(\lambda)\left[1-e^{-\tau_{1}(\lambda)\left(t-t_{0}\right)}\right]-A_{2}(\lambda)\left[1-e^{-\tau_{2}(\lambda)\left(t-t_{0}\right)}\right] \\
& \frac{1}{K_{r c}\left(\lambda_{3-8}, t\right)}=A_{0}(\lambda)-A_{1}(\lambda)\left[1-e^{-\tau(\lambda)\left(t-t_{0}\right)}\right]-A_{2}(\lambda)\left(t-t_{0}\right)
\end{aligned}
$$

where:

$$
\begin{aligned}
& A_{i} \equiv \text { fitted values of the function } \\
& \tau_{i} \equiv \text { time constants of the exponential functions } \\
& t_{0} \equiv \text { reference time for the time series. }
\end{aligned}
$$

For Bands 1 and 2, the CVT used a simultaneous exponential with a short-period time constant of 200 days and a long-period time constant of 3200 days to fit the radiometric response of the instrument (using Equation 2). For Bands 3-8, the CVT used the 400-day exponential plus linear function of time (using Equation 3). The SeaWiFS calibration tables incorporate the coefficients $A_{i}$ and the time constants $\tau_{i}$ of the fitting functions.

The fully calibrated lunar data have the long-term radiometric corrections applied and, ideally, should be constant over time:

$$
\begin{aligned}
L_{F C m n}(\lambda, t) & =L_{M o o n}(\lambda, t) K_{r c}(\lambda, t) K_{v g}(\lambda, t) K_{o s}(\lambda, t) \\
L_{F C m}(\lambda, t) & =L_{\text {Moon }}(\lambda, t) K_{r c}(\lambda, t) K_{v g}(\lambda, t) K_{o s}(\lambda, t) K_{c n}(\lambda, t)
\end{aligned}
$$

where Equation 5 has the coherent noise correction applied and where:

$$
\begin{array}{lll}
L_{M o o n} & \equiv \text { disk-integrated lunar radiances } \\
K_{r c} & \equiv \text { long-term radiometric correction } \\
K_{v g} & \equiv \text { viewing geometry correction } \\
K_{o s} & \equiv \text { oversampling correction } \\
K_{c n} & \equiv \text { coherent noise correction. }
\end{array}
$$



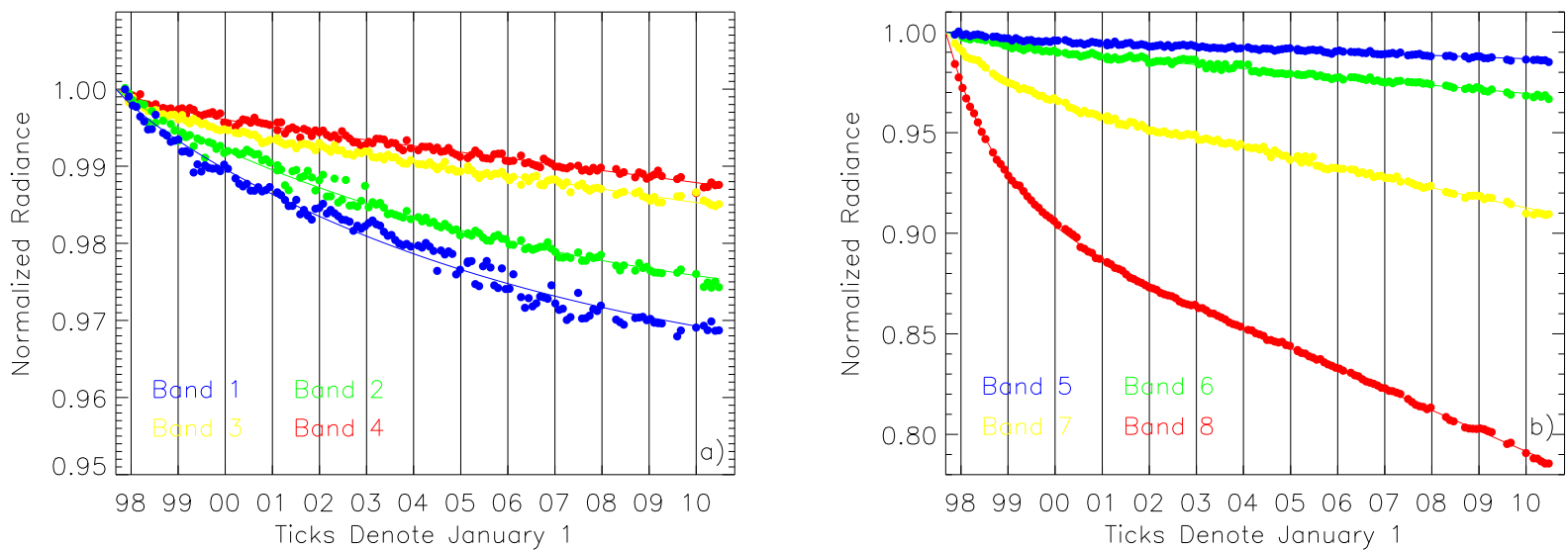

Figure 1. Lunar Calibration Time Series. a) The lunar time series for bands 1-4, with long-term radiometric fits. b) The lunar time series for bands 5-8, with long-term radiometric fits.
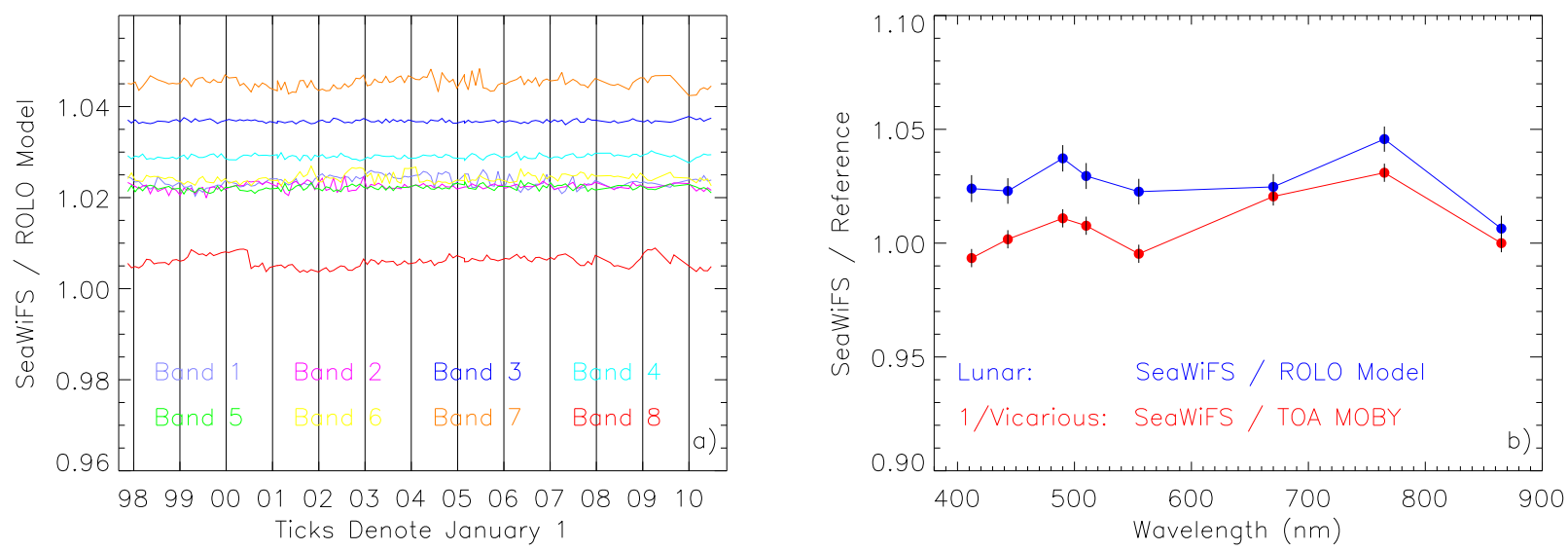

Figure 2. SeaWiFS On-Orbit Calibrations. a) The long-term stability of the on-orbit calibration of SeaWiFS is shown for each band. b) The mission-averaged lunar data comparison with the ROLO model (shown in blue) and the vicarious calibration comparison with MOBY (shown in red) are shown as a function of wavelength. The error bars for the lunar data are the RMS errors of the time series and the error bars for the vicarious gains are the uncertainties in the gains of 0.001 .

The time series including the coherent noise correction are shown in Fig. 2. The measured uncertainties in $L_{F C m}$ arise from the RMS error in the radiances and from the calibration biases. The uncertainties from the lunar calibration time series that are applicable to Earth observations are those due to the long-term radiometric correction. Hence, the CVT minimizes the systematic errors in the lunar observations that are not present in the Earth observations, namely the uncertainties from the oversampling corrections and the viewing geometry corrections, through the coherent noise correction. The derivation of the coherent noise correction is the subject of the next section of the paper.

\subsubsection{Coherent Noise Correction for the Lunar Data}

The fully-calibrated lunar time series for Bands $1(412 \mathrm{~nm})$ and $5(555 \mathrm{~nm})$, without the coherent noise correction, are shown in Fig. 3a. The scatter in the time series arises largely from uncertainties in the oversampling correction, along with contributions from uncertainties in the viewing geometry corrections. ${ }^{12}$ The oversampling correction is derived from the determination of the size of the lunar image in the along-track direction, so the $\sim \frac{1}{4}$ pixel uncertainty in the size of the Moon in a given lunar image results in an $\sim 2 \%$ uncertainty in the oversampling correction. Since the same oversampling correction is applied to each band, the errors in the correction are 


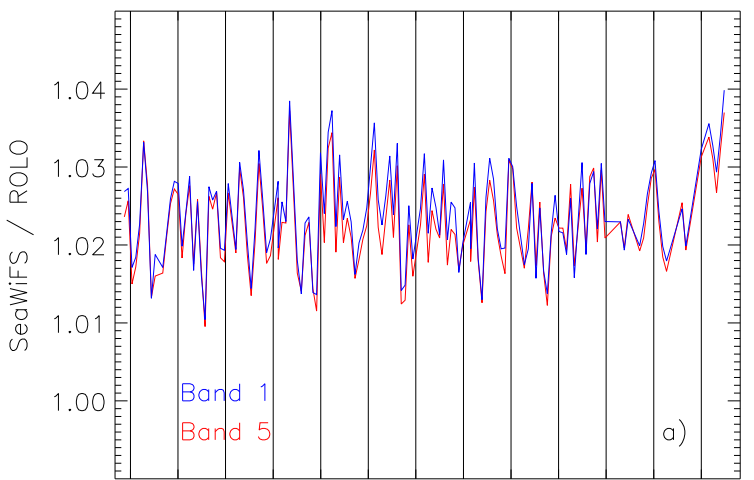

98990001020304050607080910 Ticks Denote January 1

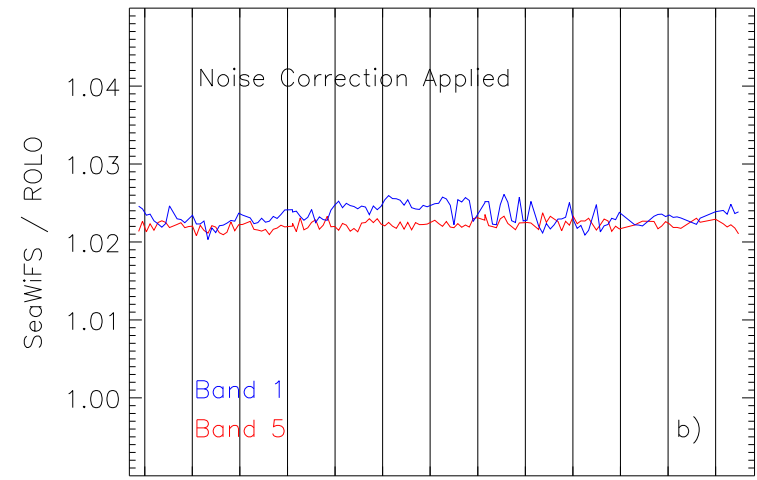

98990001020304050607080910 Ticks Denote January 1

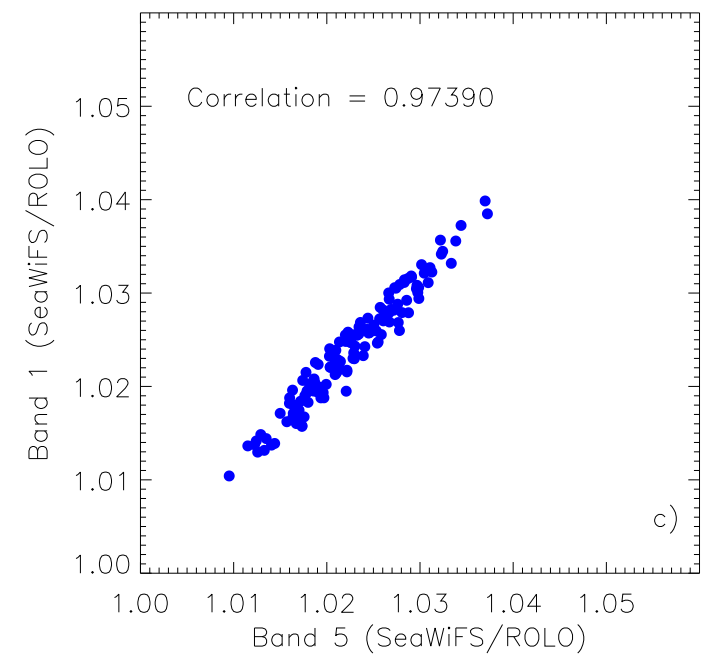

Figure 3. Correlated Band 1 / Band 5 Lunar Residuals. a) The time series of the fully-calibrated lunar observations for Bands 1 and 5. b) The lunar time series with the coherent noise correction applied shows the long-term stability of the radiometric calibration of the instrument. c) The plot of the lunar residuals for the two bands shows the correlation of the errors.

correlated between bands. The coherence in the scatter between the two bands is shown by the high correlation in the residuals plotted in Fig. 3c. Since Band 5 exhibits the smallest change in radiometric response over time, the CVT has used this band as the reference band for evaluating noise correlations among all eight bands. Table 2 shows that the noise in the SeaWiFS bands is correlated at the level of $96-99 \%$. The CVT has used the band-to-band correlation of this noise to implement a coherent noise correction for the lunar time series. ${ }^{13,14}$

An estimate of the coherent noise in each band can be determined by computing the residuals of a fit $F$ over time to the lunar time series that does not have the coherent noise correction applied: ${ }^{14}$

$$
R_{c n}(\lambda, t)=\frac{L_{M o o n}(\lambda, t)-F(\lambda, t)}{F(\lambda, t)}
$$

The coherent noise estimates can be contaminated by changes in radiometric response over time, so the CVT has found that for SeaWiFS the most reliable coherent noise estimates come from the bands that have minimal changes in response with time, Bands 3-5. The high level of correlation in the noise among the SeaWiFS bands has allowed the CVT to implement a correction to mitigate the coherent noise in the lunar time series:

$$
K_{c n}(t)=1-\left\langle R_{c n}(\lambda, t)\right\rangle_{\lambda}
$$


Table 2. Correlated Lunar Residuals. The correlation between Band 5 and the remaining bands, ${ }^{b}$ before and ${ }^{a}$ after the coherent noise correction.

\begin{tabular}{||c|c||c|c||}
\hline Bands & $\lambda(\mathbf{n m})$ & Correlation $^{b}$ & Correlation $^{a}$ \\
\hline \hline $1 / 5$ & $412 / 555$ & 0.97390 & 0.03447 \\
\hline $2 / 5$ & $443 / 555$ & 0.98441 & 0.04884 \\
\hline $3 / 5$ & $490 / 555$ & 0.98999 & 0.55101 \\
\hline $4 / 5$ & $510 / 555$ & 0.98605 & 0.67144 \\
\hline $6 / 5$ & $670 / 555$ & 0.98946 & 0.52583 \\
\hline $7 / 5$ & $765 / 555$ & 0.97762 & 0.16782 \\
\hline $8 / 5$ & $865 / 555$ & 0.96665 & 0.08396 \\
\hline
\end{tabular}

where the noise estimates $\left\langle R_{c n}(\lambda, t)\right\rangle_{\lambda}$ are averaged over Bands $3-5$. This single coherent noise correction is applied to all eight bands. Table 2 shows that, after the coherent noise correction has been applied, the correlations in the noise between the bands have been reduced by factors of $2-20$. The noise correction must not change any time dependence in the radiometric response of the band to which it is applied, so the same fitting function that is used to compute the long-term radiometric correction for the time series is used to compute the noise estimate, an exponential plus linear function or simultaneous exponential functions. For the fully-calibrated lunar data with the long-term radiometric correction applied the fitting function is a linear function time, since any residual radiometric drift in a given band should be small. Fig. 3b shows Bands 1 and 5 with the coherent noise correction applied. The residual time dependence for each of the bands is the same with or without the noise correction, which verifies the time independence of the noise correction.

Since the viewing geometry corrections and the oversampling correction only apply to the lunar data, the coherent noise correction $K_{c n}$ mitigates the systematic errors found in $K_{v g}$ and $K_{o s}$, thus minimizing the systematic errors in the long-term radiometric correction $K_{r c}$ that are applied to the Earth data. By doing so, the coherent noise correction allows the uncertainties derived for the lunar time series (with the coherent noise correction) to be applied to the Earth data that is calibrated with the radiometric correction.

\subsection{Solar Data}

The solar calibration time series for Band 1 is shown in Fig. 4. The orbit node of the SeaWiFS spacecraft drifted over the mission, giving rise to a temporal trend in the solar $\beta$ angle. Since the bidirectional reflectance distribution function (BRDF) of the solar diffuser was only measured (incompletely) for Bands 4 and 8 during the prelaunch instrument characterization, and since SeaWiFS does not have a means of measuring the diffuser BRDF on orbit, the CVT has developed a set of empirical corrections to the solar time series for the effects of the $\beta$ angle variation, the orbit node drift, and the solar diffuser degradation. The fully calibrated solar data have the long-term radiometric corrections, the Earth-Sun distance corrections, and the solar $\beta$-angle corrections, and the solar diffuser reflectance degradations applied applied:

$$
L_{F C s}(\lambda, t)=L_{\text {Solar }}(\lambda, t) K_{r c}(\lambda, t) K_{e s}(t) K_{\beta}(\lambda, t) K_{\text {diff }}(\lambda, t)
$$

where:

$$
\begin{array}{ll}
L_{\text {Solar }} & \equiv L_{T} \text { for solar diffuser measurements } \\
K_{r c} & \equiv \text { long-term radiometric correction } \\
K_{e s} & \equiv \text { Earth-Sun distance correction } \\
K_{\beta} & \equiv \quad \text { angle correction for the position of the Sun in the sky } \\
K_{\text {diff }} \equiv \text { diffuser reflectance degradation correction. }
\end{array}
$$

The $\beta$ angle corrections were derived from multiple linear regressions of the $\beta$ angle time series and the node drift time series against the diffuser time series. The regression equation, which is the inverse of the $\beta$ angle 
correction $K_{\beta}$ is:

$$
\frac{1}{K_{\beta}(\lambda, t)}=r_{0}(\lambda)+r_{1}(\lambda) \cos (\beta(t))+r_{2}(\lambda) \sin (\beta(t))+r_{3}(\lambda) \Omega(t)
$$

where:

$$
\begin{aligned}
& r_{i} \equiv \text { regression coefficients } \\
& \beta \equiv \beta \text { angle of the Sun on the diffuser } \\
& \Omega \equiv \text { node of the satellite's orbit } \\
& \lambda \equiv \text { SeaWiFS band } \\
& t \equiv \text { time of the solar observation }
\end{aligned}
$$

For the diffuser degradation analysis, the CVT fit the solar diffuser time series, corrected for the solar $\beta$ angle, with a decaying exponential function of time for each band. These functions, which are the inverses of the diffuser reflectance correction $K_{\text {diff }}$, have the form:

$$
\frac{1}{K_{d i f f}(\lambda, t)}=a_{0}(\lambda)-a_{1}(\lambda)\left[1-e^{-a_{2}(\lambda)\left(t-t_{0}\right)}\right]
$$

where:

$$
\begin{aligned}
& a_{i} \equiv \text { fit coefficients } \\
& \lambda \equiv \text { SeaWiFS band } \\
& t \equiv \text { the time of the solar observation } \\
& t_{0} \equiv \text { the reference time for the time series }
\end{aligned}
$$

The uncertainties in the combined corrections for the $\beta$ angle and the diffuser reflectances are $\sim 2 \% .{ }^{15}$

The fully-calibrated solar time series provides a unique on orbit instrumentation capability to the CVT: a uniformly-illuminated, stable, full-aperture radiance source for SeaWiFS. These uniformly-illuminated, fullaperture data allow the CVT to calculate the signal-to-noise ratio (SNR) for the instrument on orbit:

$$
\operatorname{SNR}(\lambda, t)=\frac{<L_{F C s}(\lambda, t)>}{\sigma\left(<L_{F C s}(\lambda, t)>\right)}
$$

where the standard deviations of the mean radiances for the individual diffuser observations serve as the noise estimates. These noise estimates are stable over the mission with changes of less than one count for each band. ${ }^{15}$ The signal-to-noise ratios for SeaWiFS are summarized in Table 3, which provides the prelaunch SNRs computed for typical TOA radiances and the SNRs measured on-orbit from the solar calibration time series. The prelaunch and on-orbit SNRs are computed for different mean radiances, so the measured SNRs are compared to the output of an empirical sensor noise model. Examination of the computed SNRs shows that there have not been appreciable changes in the SeaWiFS SNRs between the prelaunch and on-orbit determinations.

\subsection{Earth Data}

The calibration of the Earth data applies the long-term radiometric correction derived from the lunar calibration time series to TOA radiances, with a further correction applied to Band 7 for the Gain 3 drift:

$$
\begin{aligned}
L_{F C e}\left(\lambda_{1-6,8}, t\right) & =L_{T}(\lambda, t) K_{r c}(\lambda, t) \\
L_{F C e}\left(\lambda_{7}, t\right) & =L_{T}\left(\lambda_{7}, t\right) K_{r c}\left(\lambda_{7}, t\right) K_{g 3}\left(\lambda_{7}, t\right)
\end{aligned}
$$

where:

$$
\begin{aligned}
& L_{T} \equiv \text { top-of-the-atmosphere radiance. } \\
& K_{r c} \equiv \text { long-term radiometric correction. } \\
& K_{g 3} \equiv \text { Gain } 3 \text { drift correction (for Band } 7 \text { Earth data only). }
\end{aligned}
$$

The uncertainties in $L_{F C e}$ arise from the RMS error in the radiances, from the calibration biases, from the long-term stability of radiometric correction, and for Band 7, from the uncertainty in the Gain 3 drift correction. The derivation of the Gain 3 drift correction is the subject of the next section of the paper. 

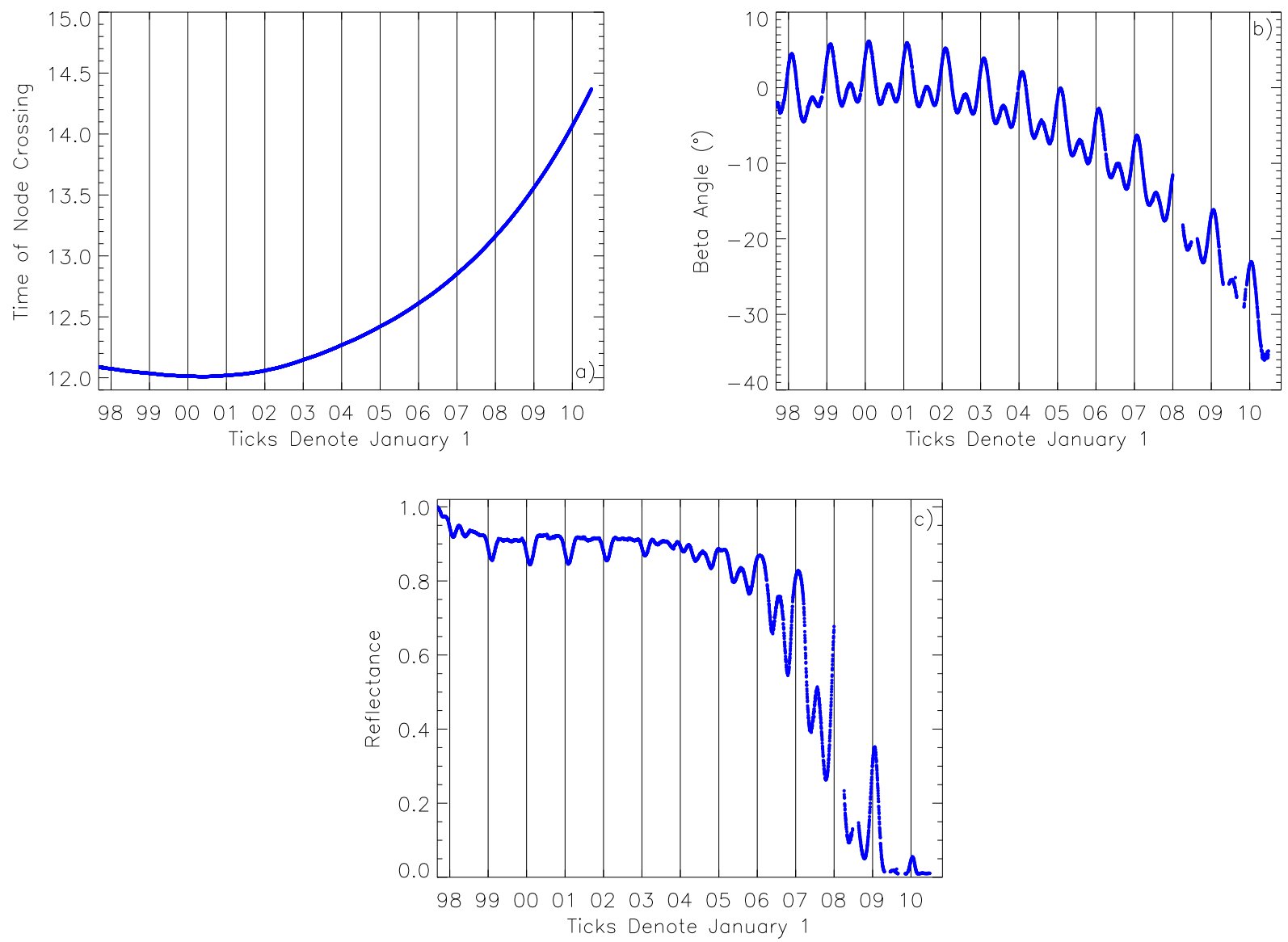

Figure 4. Solar Calibration Time Series. a) The orbit node has drifted in time from 12:00 noon to 2:20 pm. b) The beta angle time series shows the temporal trend of the orbit node drift. c) The Band 1 time series (with the long-term radiometric correction applied) shows the combined effects of the solar $\beta$ angle variation, the orbit node drift, and the diffuser reflectance degradation.

Table 3. SeaWiFS Signal-to-Noise Ratios. The prelaunch SNRs are measured at $L_{t y p}$, while the on-orbit SNRs are measured at $\left\langle L_{F C s}\right\rangle$. The radiance units are $m W \mathrm{~cm}^{-2} \mu \mathrm{m}^{-1} \mathrm{sr}^{-1}$. The table also shows the SNR specification for each band

\begin{tabular}{||c|c||c|c|c|c||c|c|c||}
\hline \multicolumn{2}{||c||}{} & \multicolumn{4}{c||}{ Prelaunch } & \multicolumn{3}{c||}{ On Orbit } \\
\hline \hline Band & $\lambda(\mathbf{u m})$ & $\mathbf{L}_{\text {typ }}$ & $\begin{array}{c}\text { SNR } \\
\text { Spec }\end{array}$ & $\begin{array}{c}\text { Model } \\
\text { SNR }\end{array}$ & $\begin{array}{c}\text { Measured } \\
\text { SNR }\end{array}$ & $<$ LFCs $_{\text {FN }}$ & $\begin{array}{c}\text { Model } \\
\text { SNR }\end{array}$ & $\begin{array}{c}\text { Measured } \\
\text { SNR }\end{array}$ \\
\hline \hline 1 & 412 & 9.10 & 499 & 990 & 940 & 4.44 & 736 & 646 \\
\hline 2 & 443 & 8.41 & 674 & 1091 & 950 & 5.19 & 784 & 794 \\
\hline 3 & 490 & 6.56 & 667 & 1170 & 1156 & 5.45 & 955 & 976 \\
\hline 4 & 510 & 5.64 & 616 & 1152 & 1055 & 5.33 & 943 & 1013 \\
\hline 5 & 555 & 4.57 & 581 & 1069 & 963 & 5.18 & 859 & 953 \\
\hline 6 & 670 & 2.46 & 447 & 781 & 798 & 4.43 & 572 & 833 \\
\hline 7 & 765 & 1.61 & 455 & 859 & 860 & 3.63 & 668 & 857 \\
\hline 8 & 865 & 1.09 & 467 & 726 & 670 & 2.99 & 557 & 767 \\
\hline
\end{tabular}


Table 4. Commanded Gains. Gain 1 is the standard gain for ocean observations. Gain 2 is the secondary gain (2x) for ocean observations. Gain 3 and Gain 4 are the commanded gains designed to give 3/4 full scale output for lunar and solar observations.

\begin{tabular}{|c|c|c|c|}
\hline Target & Band 1 & Band 2 & Bands 3-8 \\
\hline ocean & Gain 1 & Gain 1 & Gain 1 \\
lunar & Gain 4 & Gain 3 & Gain 3 \\
solar & Gain 3 & Gain 1 & Gain 3 \\
2X & Gain 2 & Gain 2 & Gain 2 \\
\hline
\end{tabular}

\subsubsection{Gain 3 Drift Correction}

Since SeaWiFS observes the Moon at a different set of instrument gains than those used for ocean observations (see Table 4), the radiometric trends derived from lunar observations are applicable to the ocean data as long as the gain ratios are constant or as long as any drifts in the gain ratios are corrected in the ocean data. The SeaWiFS on-orbit gain calibration uses a constant voltage source (the calibration pulse), injected into the postdetector electronics, to monitor output of the electronic gains over time. ${ }^{15,16}$ The gain calibration data analysis generates time series of gain ratios for each band. The gain ratios (GR1, GR2, GR3, GR4) for a given band are defined relative to Gain 1 as follows:

$$
G R X(\lambda, t)=\frac{C P(G X, \lambda, t)}{C P(G 1, \lambda, t)}
$$

where X designates the commanded gain (Gain 1, Gain 2, Gain 3, or Gain 4) and CP is the output counts from the band for a commanded gain with the calibration pulse as input. The CVT examined the gain ratio time series for possible trends. The mission-averaged on-orbit gain ratios, computed for each band and detector from the calibration pulse time series, agree with the on-orbit gain ratios computed by Eplee et al. (2007). ${ }^{15}$

The gain ratios for the commanded lunar gains over the SeaWiFS mission are given in Table 5. The table shows that there is no drift in the gain ratios over the mission for Bands 1-5, a minimal drift for Band 6 , a significant drift for Band 7, and a marginally significant drift for Band 8. This analysis is limited by the digitization of the calibration pulse data as it comes out of the instrument electronics, which preferentially affects the longer-wavelength bands. The Band 7, Gain 3 time series is the only gain ratio in all 8 bands that is out of family with the other gain ratios in that band; the Band 7 time series are shown in Fig. 5. The CVT has incorporated a correction for the Band 7 Gain 3 drift into the instrument calibration. The Band 8 Gain 3 drift is almost large enough to warrant a correction, as well, but the large quantization error for Band 8 precludes a useful correction from being computed. The form of the Gain 3 drift, which is the inverse of the drift correction $K_{g 3}$ that is applied to the Band 7 Earth data, is a quadratic function of time:

$$
\frac{1}{K_{g 3}\left(\lambda_{7}, t\right)}=A_{0}\left(\lambda_{7}\right)+A_{1}\left(\lambda_{7}\right)\left(t-t_{0}\right)+A_{2}\left(\lambda_{7}\right)\left(t-t_{0}\right)^{2}
$$

where $A_{i}$ are the fit coefficients.

The uncertainties in the gain ratio trends over time arise from actual changes in the instrument gains, instability in the calibration pulse output, and quantization error in the red bands. These uncertainties only apply to the Earth data for Band 7, where the gain drift correction is applied. The Band 7 Gain 3 drift correction reduces the uncertainty in the gain ratio trending from $0.201 \%$ to $0.148 \%$.

\subsection{Vicarious Calibration of Earth Data}

Addressing specific scientific questions about the Earth's climate based on satellite observations of the Earth requires that the TOA radiances of the remote sensing instruments be vicariously calibrated to yield accurate results at the surface of interest. For example, the vicarious calibration of ocean color data from Earth remote sensing instruments adjusts the on-orbit calibration of the instruments to match the system-level calibration of 
Table 5. Lunar Gain Ratios. The gain ratios for lunar calibrations are given for each band. The calibration pulse counts from which the ratios were derived are provided for the start and end of the missions. The long-term stability of the gain ratios are provided. ${ }^{*}$ The band 7 stability is also provided after the Gain 3 drift correction.

\begin{tabular}{||c|c||c|c||c|c||c|}
\hline Band & $\begin{array}{c}\lambda \\
\text { nm }\end{array}$ & Gain & $\begin{array}{c}\text { Gain } \\
\text { Ratio }\end{array}$ & $\begin{array}{c}\text { Initial } \\
\text { Counts }\end{array}$ & $\begin{array}{c}\text { Final } \\
\text { Counts }\end{array}$ & $\begin{array}{c}\text { RMS } \\
\text { Error (\%) }\end{array}$ \\
\hline \hline 1 & 412 & 4 & 1.597 & 614 & 608 & 0.0598 \\
\hline 2 & 443 & 3 & 1.292 & 492 & 478 & 0.0602 \\
\hline 3 & 490 & 3 & 0.9010 & 329 & 324 & 0.0732 \\
\hline 4 & 510 & 3 & 0.7975 & 290 & 282 & 0.103 \\
\hline 5 & 555 & 3 & 0.6582 & 239 & 233 & 0.110 \\
\hline 6 & 670 & 3 & 0.4016 & 145 & 143 & 0.143 \\
\hline 7 & 765 & 3 & 0.3393 & 119 & 117 & 0.201 \\
& & & & & & $0.148^{*}$ \\
\hline 8 & 865 & 3 & 0.2942 & 109 & 108 & 0.174 \\
\hline
\end{tabular}
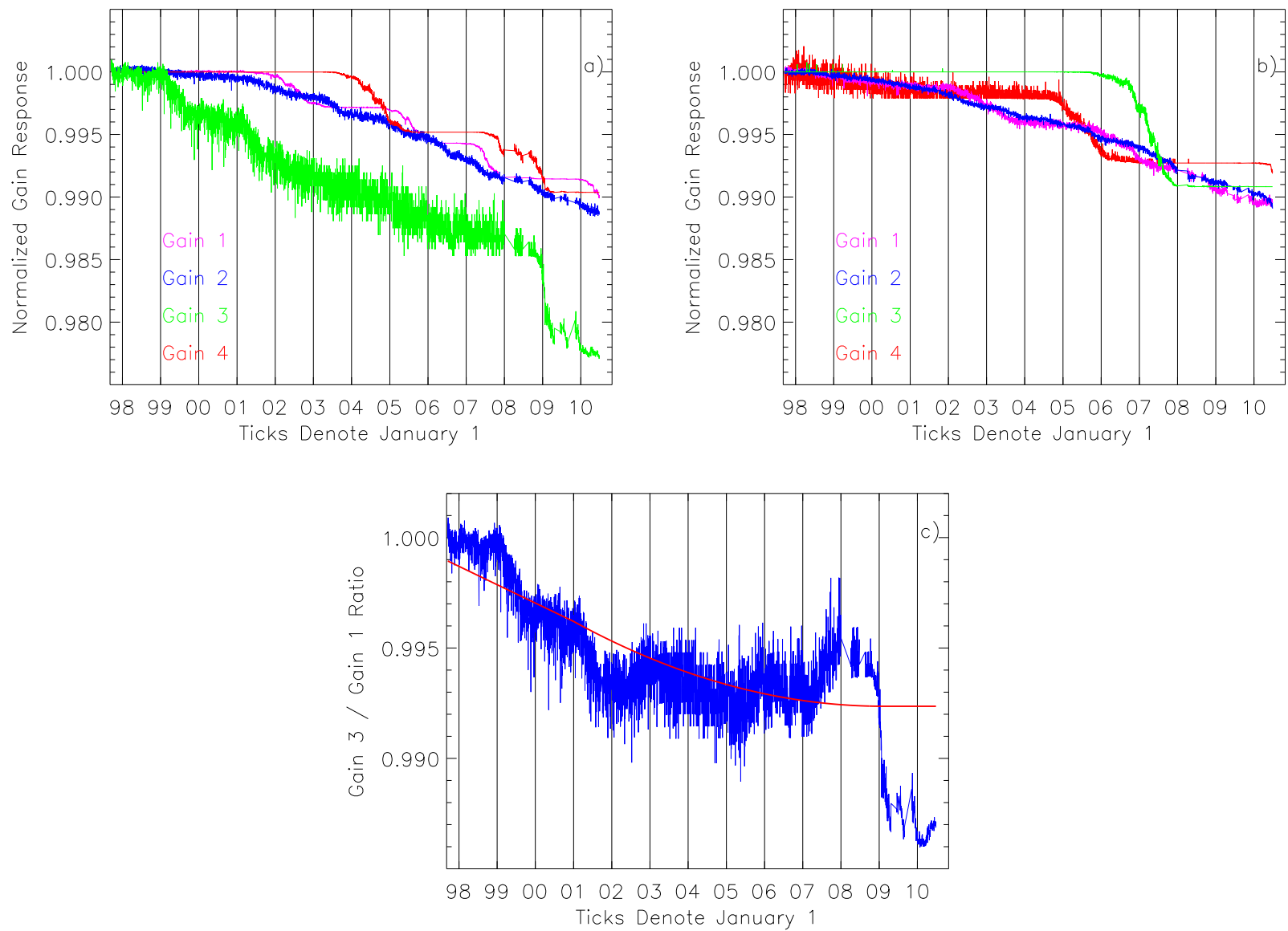

Figure 5. Band 7 and 8 Gain Calibrations. a) The Band 7 time series; Gain 3 is the only time series in all eight bands that is out of family with the other gains in that band. b) The Band 8 time series; Gain 3 is impacted by digitization noise. c) The Band 7 Gain 3/Gain 1 drift correction for the ocean color data. 
the in situ radiometer and atmospheric correction algorithm. ${ }^{2}$ The vicarious calibration mitigates uncertainties (e.g., biases) in the calibration of the satellite instrument, the calibration of the in situ radiometer, and the atmospheric correction algorithm. By adjusting the calibration of the SeaWiFS TOA radiances (with the long-term radiometric correction already applied) so that the retrieved SeaWiFS water-leaving radiances match corresponding in-water measurements from the Marine Optical Buoy (MOBY), the vicarious calibration provides a means of cross calibrating SeaWiFS with the MOBY / atmospheric correction algorithm. As another example, atmospheric data products may not require any vicarious calibration of the TOA radiances.

The vicarious calibration procedure for ocean color data from SeaWiFS is as follows:

1) The calibration of Band $8(865 \mathrm{~nm})$ is assumed to be correct. The Band 8 gain can be off by $\sim 5 \%$ without introducing a significant error into the vicarious calibration. ${ }^{17,18}$

2) Band $7(765 \mathrm{~nm})$ is calibrated relative to Band 8 so the atmospheric correction algorithm retrieves the expected aerosol types and optical depths for open ocean scenes.

3) The TOA radiances computed for the visible bands are calibrated against in-water measurements from MOBY, propagated to the top of the atmosphere using the retrieved atmospheric correction parameters.

Vicarious calibration should yield the optimum water-leaving radiances for the sensor/algorithm system-level calibration. The vicarious gains for bands 1-6 are the mission-long averages of the ratio of the in-water radiances measured by MOBY and propagated to the top of the atmosphere to the TOA radiances measured by SeaWiFS: ${ }^{2}$

$$
K_{v c}(\lambda, t)=\left\langle\frac{L_{w}(\lambda, t) t_{w}(\lambda, t)+L_{f}(\lambda, t) t_{f}(\lambda, t)+L_{r}(\lambda, t) t_{r}(\lambda, t)+L_{a}(\lambda, t) t_{a}(\lambda, t)}{L_{T}(\lambda, t)}\right\rangle
$$

where:

$L_{w} \equiv$ in-water radiance measured by MOBY

$L_{f} \equiv$ sea foam and whitecap radiance

$L_{r} \quad \equiv$ Rayleigh radiance

$L_{a} \equiv$ aerosol radiance

$L_{T} \equiv$ TOA radiance measured by SeaWiFS

$t_{i} \equiv$ atmospheric transmission for radiance component $i$

The vicariously calibrated TOA radiances are:

$$
\begin{aligned}
L_{V C e}\left(\lambda_{1-6}, t\right) & =L_{T}(\lambda, t) K_{r c}(\lambda, t) K_{v c}(\lambda) \\
L_{V C e}\left(\lambda_{7}, t\right) & =L_{T}\left(\lambda_{7}, t\right) K_{r c}\left(\lambda_{7}, t\right) K_{g 3}\left(\lambda_{7}, t\right) K_{v c}\left(\lambda_{7}\right) \\
L_{V C e}\left(\lambda_{8}, t\right) & =L_{T}\left(\lambda_{8}, t\right) K_{r c}\left(\lambda_{8}, t\right)
\end{aligned}
$$

where $K_{v c} \equiv$ vicarious gains (for Bands 1-7 Earth data only). The uncertainty in calibrated radiances $L_{V C e}$ arises from the RMS error in the radiances, the calibration biases for SeaWiFS, the uncertainty in the Gain 3 drift correction (for Band 7 only), and the uncertainty in the vicarious calibration (for Bands 1-7). The uncertainty in the vicarious calibration itself has two sources: the vicarious gains and the water-leaving radiances measured by MOBY, then propagated to the top of the atmosphere. The uncertainty in the vicarious gains arises from the uncertainty in the atmospheric correction. ${ }^{3,19}$ The uncertainties for individual vicarious calibration data points are up to $\sim 1 \%$, but with the collection of 20-40 data points the vicarious gains have converged to values that have uncertainties of $0.1 \% .^{2}$ The uncertainty in the water-leaving radiances is $2.1-3.3 \%$ (depending on the band), which becomes $0.21-0.33 \%$ when propagated to the top of the atmosphere. ${ }^{20}$ Table 6 shows the vicarious calibration used in the 2010 reprocessing of the SeaWiFS global ocean color data set.

\section{UNCERTAINTY IN THE TOA RADIANCES}

Having reviewed the sources of uncertainty in the SeaWiFS on-orbit calibration, this paper will now merge the various uncertainty estimates to present the overall uncertainty in the calibrated TOA radiances. Again, the uncertainty will be addressed in the terms of accuracy or calibration biases, stability or repeatability of the measurements over time, and precision or scatter in the measurements. 
Table 6. Vicarious Calibration. ${ }^{+}$Gains derived from MOBY radiances propagated to the TOA. \# Gains derived from aerosol retrievals. ${ }^{*}$ No vicarious calibration. The MOBY uncertainties are for the water-leaving radiances.

\begin{tabular}{||c|c||c|c|c||c|}
\hline Band & $\lambda(\mathbf{n m})$ & $\begin{array}{c}\text { Vicarious } \\
\text { Gains }\end{array}$ & $\begin{array}{c}\text { Calibration } \\
\text { Bias (\%) }\end{array}$ & $\begin{array}{c}\text { Gain } \\
\text { Uncertainty (\%) }\end{array}$ & $\begin{array}{c}\text { MOBY } \\
\text { Uncertainty (\%) }\end{array}$ \\
\hline \hline 1 & 412 & $1.0066^{+}$ & -0.656 & 0.07 & 2.4 \\
\hline 2 & 443 & $0.9983^{+}$ & 0.170 & 0.07 & 2.1 \\
\hline 3 & 490 & $0.9892^{+}$ & 1.09 & 0.07 & 2.4 \\
\hline 4 & 510 & $0.9924^{+}$ & 0.766 & 0.07 & 2.3 \\
\hline 5 & 555 & $1.0047^{+}$ & -0.468 & 0.07 & 2.4 \\
\hline 6 & 670 & $0.9799^{+}$ & 2.05 & 0.06 & 3.3 \\
\hline 7 & 765 & $0.9700^{\#}$ & 3.09 & 0.11 & \\
\hline 8 & 865 & $1.00^{*}$ & & & \\
\hline
\end{tabular}

\subsection{Accuracy of the TOA Radiances}

The mission-averaged mean lunar residuals from the ROLO model and the magnitude of the vicarious calibration gains provide the best on-orbit estimates of the biases in the SeaWiFS TOA radiances that are internal to the SeaWiFS calibration. The measurement accuracy is defined as:

$$
\text { Accuracy }=\left\langle\frac{\text { SeaWiFS }}{\text { Reference }}-1\right\rangle
$$

so the accuracy of the vicarious calibration is derived from the inverses of the vicarious gains, as shown by Equation 15. Fig. 2b shows the mean ROLO residuals for the lunar observations and the inverses of the vicarious gains computed relative to MOBY. The trends in the biases with wavelength are comparable, though the biases are smaller for the vicarious calibration. The biases in the SeaWiFS TOA radiances are shown in Table 7. The average bias in the lunar calibration is $2.62 \pm 1.16 \%$. The average bias in the vicarious calibration for Bands $1-7$ is $1.19 \pm 1.03 \%$. In summary, the accuracy of the SeaWiFS TOA radiances is $2-3 \%$ relative to the ROLO model or 1-2\% relative to MOBY. For comparison purposes, the uncertainty in the NIST prelaunch calibration of SeaWiFS is $4 \%,{ }^{10}$ and the cross calibration of SeaWiFS relative to Terra MODIS and Aqua MODIS on orbit is $3-8 \%{ }^{12}$ The measured accuracy of the SeaWiFS TOA radiances is with the uncertainties in the prelaunch calibration. The key question that remains unanswered in the accuracy or bias determination is one of bias compared to which external reference? The specific scientific question being addressed determines the external reference for the calibration bias.

\subsection{Long-Term Stability of the TOA Radiances}

The long-term stability of the TOA radiances is a validation of the effectiveness of the radiometric correction $K_{r c}$ and is a measure of the degree to which a single-point vicarious calibration is applicable to the Earth data. The uncertainty in the long-term stability arises from any residual time dependence in the TOA radiances. Accordingly, the limits on the residual time drift in the fully-calibrated lunar time series provide the best estimate on-orbit of the long-term stability of the SeaWiFS TOA radiances. The standard deviation of the mean (or RMS error) of the mission-averaged lunar time series for each band defines the upper limit on the residual time drift in that band, so the actual calibration stability could be better than the RMS error.

As has been discussed previously in this paper, systematic errors in the lunar observations that are not present in the Earth observations, namely the uncertainties from the oversampling corrections and the viewing geometry corrections, give rise to errors in the lunar data that are correlated from band to band, and as such, can be mitigated through the coherent noise correction. The coherent noise correction is necessary to show the 
Table 7. On-Orbit Measurement Accuracy. The third column shows the SeaWiFS bias relative to the ROLO model. The fourth column shows the SeaWiFS bias relative to MOBY.

\begin{tabular}{||c|c||c|c|}
\hline Band & $\lambda(\mathbf{n m})$ & $\begin{array}{c}\text { SeaWiFS/ROLO } \\
\text { Bias (\%) }\end{array}$ & $\begin{array}{c}\text { SeaWiFS/MOBY } \\
\text { Bias (\%) }\end{array}$ \\
\hline \hline 1 & 412 & $2.35 \pm 0.12$ & $-0.656 \pm 0.07$ \\
\hline 2 & 443 & $2.25 \pm 0.078$ & $0.170 \pm 0.07$ \\
\hline 3 & 490 & $3.68 \pm 0.033$ & $1.09 \pm 0.07$ \\
\hline 4 & 510 & $2.90 \pm 0.046$ & $0.766 \pm 0.07$ \\
\hline 5 & 555 & $2.22 \pm 0.058$ & $-0.468 \pm 0.07$ \\
\hline 6 & 670 & $2.43 \pm 0.096$ & $2.05 \pm 0.06$ \\
\hline 7 & 765 & $4.52 \pm 0.12$ & $3.09 \pm 0.11$ \\
\hline 8 & 865 & $0.60 \pm 0.13$ & \\
\hline
\end{tabular}

true instrumental scatter in the lunar radiances. Figure 1a shows the fully-calibrated lunar time series with the coherent noise correction applied. The long-term stability (or RMS error) in the lunar time series, before and after coherent noise correction has been applied, is shown in Table 8. Similar RMS errors in each band $(\sim 0.570 \%)$ before the noise correction are indicative of the coherence in the noise between bands. The reduction in the coherent noise is optimized toward the bands used in computing the noise corrector, so a long-term stability of $0.13 \%$ for the SeaWiFS calibration is a reasonable assessment of the instrument performance across all bands. Because of the Band 7 Gain 3 drift, the long-term stability for Band 7 combines the RMS error from the lunar time series $(0.116 \%)$ with the RMS error in the gain drift correction $(0.148 \%)$ to yield a long-term stability for the TOA radiances of $0.19 \%$. It should be noted that the coherent noise correction improves the CVT's ability to compute the long-term stability of the TOA radiances by a factor of $\sim 5$.

The long-term stability of the vicariously-calibrated TOA radiances that are input to the ocean color data combines the uncertainty in the lunar-derived radiometric correction, the uncertainty in the vicarious gains (or atmospheric correction), and the uncertainty in the water-leaving radiances measured by MOBY (then propagated to the top of the atmosphere). As shown in Table 9, the long-term stability becomes the root-sumsquare (RSS) of these three uncertainties: the radiometric correction (from Table 8), the vicarious gains (from Table ??), and the water-leaving radiances at the TOA (from Table ??). For Band 7 the long-term stability combines the uncertainty of the radiometric correction, the uncertainty in the Gain 3 drift correction, and the uncertainty in the vicarious gain of Band 7. There is no vicarious calibration for Band 8 . Accordingly, the long-term stability of the vicariously-calibrated TOA radiances for Bands $1-6$ is $0.30 \%$ and for Band 7 is $0.22 \%$. It should be noted that the dominant term in the long-term stability in the vicariously-calibrated TOA radiances arises from the vicarious calibration data source, MOBY.

\subsection{Precision of the TOA Radiances}

The scatter in the on-orbit measurements made by SeaWiFS gives the precision of the TOA radiances. The signal-to-noise ratios determined from the solar diffuser measurements, and the scatter in the lunar residuals, with the coherent noise correction applied, and are two estimates of the precision of the SeaWiFS on-orbit measurements. The solar diffuser SNR analysis results are shown in Table 3, while the RMS error in the lunar residuals are shown in Table 8. The corresponding precision estimates for the SeaWiFS TOA radiances are shown in Table 10. The diffuser-derived estimates of precision are the inverses of the SNR. A reasonable assessment of the precision of the solar diffuser measurements is $0.16 \%$. As has been discussed previously, a reasonable assessment of the precision of the lunar observations is $0.13 \%$. The larger value for the solar precision arises in part because the solar-derived SNR measurements were made for radiances below $L_{t y p}$. The solar-derived precision measurements are consistent with the lunar-derived precision measurements with the coherent noise 
Table 8. Long-Term Stability of the Radiometric Calibration. The RMS errors before and after the coherent noise correction. ${ }^{*}$ Bands used to compute the coherent noise correction. ${ }^{+}$Error including the Gain 3 drift uncertainty.

\begin{tabular}{||c|c||c|c|}
\hline Band & $\lambda(\mathbf{n m})$ & $\begin{array}{c}\text { RMS Error (\%) } \\
\text { Before } K_{c n}\end{array}$ & $\begin{array}{c}\text { RMS Error (\%) } \\
\text { After } K_{c n}\end{array}$ \\
\hline \hline 1 & 412 & 0.592 & 0.124 \\
\hline 2 & 443 & 0.562 & 0.0778 \\
\hline $3^{*}$ & 490 & 0.579 & 0.0334 \\
\hline $4^{*}$ & 510 & 0.570 & 0.0456 \\
\hline $5^{*}$ & 555 & 0.562 & 0.0578 \\
\hline 6 & 670 & 0.564 & 0.0958 \\
\hline 7 & 765 & 0.554 & 0.116 \\
& & & $0.188^{+}$ \\
\hline 8 & 865 & 0.567 & 0.129 \\
\hline
\end{tabular}

Table 9. Long-Term Stability of the Vicarious Calibration. The table entries are RMS errors (\%). *Error including the Gain 3 drift uncertainty.

\begin{tabular}{||c|c||c|c|c||c|}
\hline Band & $\lambda(\mathbf{n m})$ & $\begin{array}{c}\text { Radiometric } \\
\text { Correction } K_{r c}\end{array}$ & $\begin{array}{c}\text { Vicarious } \\
\text { Gain } K_{v c}\end{array}$ & $\begin{array}{c}\text { MOBY TOA } \\
\text { Radiances }\end{array}$ & $\begin{array}{c}\text { Combined } \\
\text { RMS Error }\end{array}$ \\
\hline \hline 1 & 412 & 0.124 & 0.07 & 0.24 & 0.28 \\
\hline 2 & 443 & 0.0778 & 0.07 & 0.21 & 0.24 \\
\hline 3 & 490 & 0.0334 & 0.07 & 0.24 & 0.26 \\
\hline 4 & 510 & 0.0456 & 0.07 & 0.23 & 0.25 \\
\hline 5 & 555 & 0.0578 & 0.07 & 0.24 & 0.26 \\
\hline 6 & 670 & 0.0958 & 0.06 & 0.33 & 0.35 \\
\hline 7 & 765 & $0.188^{*}$ & 0.11 & & $0.22^{*}$ \\
\hline 8 & 865 & 0.129 & & & \\
\hline
\end{tabular}


Table 10. On-Orbit Measurement Precision. The solar-derived and lunar-derived precision are instrumental properties. The vicarious gain precision is a property of the atmospheric correction algorithm.

\begin{tabular}{||c|c||c|c||c||}
\hline Band & $\lambda(\mathbf{n m})$ & $\begin{array}{c}\text { Solar-derived } \\
\text { Precision (\%) }\end{array}$ & $\begin{array}{c}\text { Lunar-derived } \\
\text { Precision (\%) }\end{array}$ & $\begin{array}{l}\text { Vicarious Gain } \\
\text { Precision (\%) }\end{array}$ \\
\hline \hline 1 & 412 & 0.155 & 0.124 & 0.07 \\
\hline 2 & 443 & 0.126 & 0.0778 & 0.07 \\
\hline 3 & 490 & 0.102 & 0.0334 & 0.07 \\
\hline 4 & 510 & 0.0987 & 0.0456 & 0.07 \\
\hline 5 & 555 & 0.105 & 0.0578 & 0.07 \\
\hline 6 & 670 & 0.120 & 0.0958 & 0.06 \\
\hline 7 & 765 & 0.117 & 0.116 & 0.11 \\
\hline 8 & 865 & 0.130 & 0.129 & \\
\hline
\end{tabular}

correction applied, verifying that the coherent noise correction does mitigate systematic errors in the lunar time series arising from the oversampling and geometry corrections. Accordingly, a precision of $0.13 \%$ for the SeaWiFS calibration is a reasonable assessment of the instrument performance across bands.

The scatter in the vicarious gains gives the precision of the vicarious calibration, as is shown in Table 10 . As was noted in the previous section, the uncertainty in the vicarious gains arises from the uncertainty in the atmospheric correction. The precision of the vicarious calibration directly determines the number of data points in the calibration required for the vicarious gains to converge. ${ }^{2}$ For the vicarious calibration of SeaWiFS against MOBY, 30 (Band 2), 40 (Band 5) and 20 (Band 7) data points were required for the gains to converge. Consequently, the CVT required 2-3 years of on-orbit data collection to vicariously calibrate SeaWiFS.

\section{DISCUSSION OF THE UNCERTAINTY ANALYSIS}

The goal of this paper has been to assess the uncertainties in the SeaWiFS top-of-the-atmosphere radiances, the radiances that are the basis of the ocean color data products. This assessment has been made in terms of accuracy (biases in the measurements), stability (repeatability of the measurements over time), and precision (scatter in the measurements). The results of this uncertainty assessment are presented in Table 11. The absolute uncertainty is computed relative to the ROLO model for the lunar calibration or relative to MOBY for the vicarious calibration. The long-term stability is provided for the TOA radiances and for the vicariouslycalibrated TOA radiances. The precision estimates are computed from solar calibrations, lunar calibrations, and the vicarious calibration. The uncertainties in the TOA radiances can be summarized as follows: The accuracy of the TOA radiances relative to the ROLO model of the Moon are 2-3\%; the accuracy of the vicariously-calibrated TOA radiances relative to MOBY are 1-2\%. The long-term stability of the TOA radiances, primarily due to the success of the radiometric correction, is $0.13 \%$. The long-term stability of the vicariously-calibrated TOA radiances, which are inputs for the ocean color data retrievals, is $0.30 \%$. The Band 7 Gain 3 drift correction yields TOA radiances for Band 7 with a long-term stability of $0.19 \%$ and yields vicariously-calibrated TOA radiances for Band 7 with a stability of $0.22 \%$. The precision of the SeaWiFS measurements is $0.13 \%$ as computed from the lunar calibrations, $0.16 \%$ as computed from the solar calibrations, and is $0.10 \%$ as computed from the vicarious calibration. This uncertainty assessment has been developed as the performance of the instrument has changed over time and as our understanding of the instrument performance has evolved as a result. ${ }^{12-14,21-23}$

From the standpoint of climate research, the most important uncertainty in the TOA radiances is the longterm stability of the calibration. A vicarious calibration that is specific to a retrieved surface product mitigates the calibration biases. For an instrument whose radiometric response changes over time, the stability shows how well the CVT understands the evolving instrument performance on orbit and how well the long-term radiometric 
Table 11. SeaWiFS TOA Uncertainty Assessment. The accuracy is relative to the ROLO model or to MOBY. The stability is given for TOA radiances and vicariously-calibrated (VC) TOA radiances. The precision estimates have the indicated sources. Band 8 does not have a vicarious calibration. ${ }^{*}$ Including the Band 7 Gain 3 drift correction.

\begin{tabular}{||c|c||c|c|c|c|c|c|c|c||c|}
\hline \hline \multicolumn{2}{|c||}{ Uncertainty } & B1 & B2 & B3 & B4 & B5 & B6 & B7 & B8 & Overall \\
\hline \hline \multirow{2}{*}{$\begin{array}{c}\text { Accuracy } \\
(\%)\end{array}$} & ROLO & 2.35 & 2.25 & 3.68 & 2.90 & 2.22 & 2.43 & 4.52 & 0.60 & $2-3$ \\
\cline { 2 - 12 } & MOBY & -0.656 & 0.170 & 1.09 & 0.766 & -0.468 & 2.05 & 3.09 & & $1-2$ \\
\hline \hline \multirow{2}{*}{$\begin{array}{c}\text { Stability } \\
(\%)\end{array}$} & TOA & 0.124 & 0.0778 & 0.0334 & 0.0456 & 0.0578 & 0.0958 & $0.188^{*}$ & 0.129 & 0.13 \\
\cline { 2 - 12 } & VC TOA & 0.28 & 0.24 & 0.26 & 0.25 & 0.26 & 0.35 & $0.22^{*}$ & & 0.30 \\
\hline \hline \multirow{3}{*}{$\begin{array}{c}\text { Precision } \\
(\%)\end{array}$} & Solar & 0.155 & 0.126 & 0.102 & 0.0987 & 0.105 & 0.120 & 0.117 & 0.130 & 0.16 \\
\cline { 2 - 11 } & Lunar & 0.124, & 0.0778 & 0.0334 & 0.0456 & 0.0578 & 0.0958 & 0.116 & 0.129 & 0.13 \\
\cline { 2 - 11 } & Vicarious & 0.070 & 0.070 & 0.070 & 0.070 & 0.070 & 0.060 & 0.11 & & 0.10 \\
\hline
\end{tabular}

correction accounts for that changing performance. For SeaWiFS, the CVT has been able to maintain the longterm stability at the level of $\sim 0.1 \%$ from the initial report of the stability at 6 years into the mission ${ }^{14}$ onwards through the end of the mission at 13 years.

The SeaWiFS uncertainty assessment shows the necessity of a mission-long calibration/validation program for climate quality data to be produced from environmental remote sensing satellite instruments. The availability of a long-term, stable, satellite-based global ocean color data set has allowed climate researchers to examine global spatial patterns of near-surface chlorophyll over decadal timescales. ${ }^{24-27}$ This is an important lesson from SeaWiFS for NASA and NOAA as the production of ocean color data products transitions from research instruments and programs (e.g., SeaWiFS and MODIS) to operational instruments and programs (e.g. VIIRS). ${ }^{28-30}$

\section{REFERENCES}

[1] C.R. McClain, W.E. Esaias, W. Barnes, B. Guenther, D. Endres, S.B. Hooker, G. Mitchell, and R. Barnes, SeaWiFS Calibration and Validation Plan, NASA Tech. Memo. 104566 3, S.B. Hooker and E.R. Firestone, eds., NASA Goddard Space Flight Center, Greenbelt, Maryland (1992).

[2] B.A. Franz, S.W. Bailey, P.J. Werdell, and C.R. McClain, "Sensor-independent approach to the vicarious calibration of satellite ocean color radiometry," Appl. Opt. 46, 5068-5082 (2007).

[3] H.R. Gordon, "Atmospheric correction of ocean color imagery in the Earth Observing System era," $J$. Geophys. Res. 102(D14), 17081-17106 (1997).

[4] H.H. Kieffer and T.C. Stone, "The spectral irradiance of the Moon," Astron. J. 129, 2887-2901 (2005).

[5] T.C. Stone and H.H. Kieffer, "Use of the Moon to support on-orbit sensor calibration for climate change measurements," in Earth Observing Systems XI, J.J. Butler and J. Xiong, eds., Proc. SPIE 6296, 62960Y (2006).

[6] T.C. Stone, "Radiometric calibration stability and inter-calibration of solar-band instruments in orbit using the Moon," in Earth Observing Systems XIII, J.J. Butler and J. Xiong, eds., Proc. SPIE 7081, 70810X (2008).

[7] D.K. Clark, H.R. Gordon, K.J. Voss, Y. Ge, W. Broenkow, and C. Trees, "Validation of atmospheric correction over the oceans," J. Geophys. Res. 99, 7293-7307 (1997).

[8] D.K. Clark, M.E. Feinholz, M.A. Yarbrough, B.C. Johnson, S.W. Brown, Y.S. Kim, and R.A. Barnes, "Overview of the radiometric calibration of MOBY", in Earth Observing Systems VI, W.L. Barnes, ed., Proc. SPIE 4483, 64-76 (2002).

[9] R.A. Barnes, A.W. Holmes, W.L. Barnes, W.E. Esaias, C.R. McClain, and T. Svitek, SeaWiFS Prelaunch Radiometric Calibration and Spectral Characterization, NASA Tech. Memo. 104566 23, S.B. Hooker, E.R. Firestone, and J.G. Acker, eds., NASA Goddard Space Flight Center, Greenbelt, Maryland (1994). 
[10] B.C. Johnson, E.E. Early, R.E. Eplee, Jr., R.A. Barnes, and R.T. Caffrey, The 1997 Prelaunch Radiometric Calibration of SeaWiFS, NASA Tech. Memo. 206892 4, S.B. Hooker and E.R. Firestone, eds., NASA Goddard Space Flight Center, Greenbelt, Maryland (1999).

[11] R.E. Eplee, Jr., G. Meister, F.S. Patt, and C.R. McClain, "The on-orbit calibration of SeaWiFS," In preparation (2012).

[12] R.E. Eplee, Jr., J.-Q. Sun, G. Meister, F.S. Patt, X. Xiong, and C.R. McClain, "Cross calibration of SeaWiFS and MODIS using on-orbit observations of the Moon," Appl. Opt. 50, 120-133 (2011).

[13] R.A. Barnes, R.E. Eplee, Jr., G.M. Schmidt, F.S. Patt, and C.R. McClain, "Calibration of SeaWiFS. I. Direct techniques," Appl. Opt. 40, 6682-6670 (2001).

[14] R.E. Eplee, Jr., R.A. Barnes, F.S. Patt, G. Meister, and C.R. McClain, "SeaWiFS lunar calibration methodology after six years on orbit," in Earth Observing Systems IX, W.L. Barnes and J.J. Butler, eds., Proc. SPIE 5542, 1-13 (2004).

[15] R.E. Eplee, Jr., F.S. Patt, B.A. Franz, S.W. Bailey, G. Meister, and C.R. McClain, "SeaWiFS on-orbit gain and detector calibrations: Effect on ocean products," Appl. Opt. 46, 6733-6750 (2007).

[16] R.E. Eplee, Jr., F.S. Patt, G. Meister, B.A. Franz, S.W. Bailey, and C.R. McClain, "The on-orbit calibration of SeaWiFS: Revised temperature and gain corrections," in Earth Observing Systems XII, J.J. Butler and J. Xiong, eds., Proc. SPIE 6677, 66770E (2007).

[17] H.R. Gordon, "In-orbit calibration strategy for ocean color sensors," Remote Sens. Environ. 63, 265-278 (1998).

[18] M. Wang and H.R. Gordon, "Calibration of ocean color scanners: how much error is acceptable in the near infrared?," Remote Sens. Environ. 82, 497-504 (2002).

[19] Z. Ahmad, B.A. Franz, C.R. McClain, E.J. Kwiatkowska, J. Werdell, E.P. Shettle, and B.N. Holben, "New aerosol models for the retrieval of aerosol optical thickness and normalized water-leaving radiances from the SeaWiFS and MODIS sensors over coastal regions and open oceans," Appl. Opt. 49, 5545-5560 (2010).

[20] S.W. Brown, S.J. Flora, M.F. Feinholz, M.A. Yarbrough, T. Houlihan, D. Peters, Y.S. Kim, J.L. Mueller, B.C. Johnson, and D.K. Clark, "The Marine Optical BuoY (MOBY) radiometric calibration and uncertainty budget for ocean color satellite sensor vicarious calibration," in Sensors, Systems, and Next Generation Satellites XI, R. Meynart, S.P. Neeck, H. Shimoda, and S. Habib, eds., Proc. SPIE 6744, 67441M (2007).

[21] R.A. Barnes, R.E. Eplee, Jr., F.S. Patt, and C.R. McClain, "Changes in the radiometric sensitivity of SeaWiFS determined from lunar and solar-based measurements," Appl. Opt. 38, 4649-4664 (1999).

[22] R.A. Barnes, R.E. Eplee, Jr., F.S. Patt, H.H. Kieffer, T.C. Stone, G. Meister, J.J. Butler, and C.R. McClain, "Comparison of SeaWiFS measurements of the Moon with the U.S. Geological Survey lunar model," Appl. Opt. 43, 5838-5854 (2004).

[23] R.E. Eplee, Jr., G. Meister, F.S. Patt, and C.R. McClain, "The on-orbit calibration of SeaWiFS: Functional fits to the lunar time series," in Earth Observing Systems XIII, J.J. Butler and J. Xiong, eds., Proc. SPIE 7081, 708112 (2008).

[24] C.R. McClain, "A decade of satellite ocean color observations," Annu. Rev. Mar. Sci. 1, 19-42 (2009).

[25] S.A. Henson, J.L. Sarmiento, J.P. Dunne, L. Bopp, I. Lima, S.C. Doney, J. John, and C. Beaulieu, "Detection of anthropogenic climate change n satellite records of ocean chlorophyll and productivity," Biogeosciences 7, 621-640 (2010).

[26] S. Maritorena, O.H.F. d'Andon, A. Mangin, and D.A. Siegel, "Merged satellite ocean color data products using a bio-optical model: Characteristics, benefits and issues," Rem. Sens. Environ. 114, 1791-1804 (2010).

[27] F.P. Chavez, M. Messie, and J.T. Pennington, "Marine primary production in relation to climate variability and change," Annu. Rev. Mar. Sci. 3, 227-260 (2011).

[28] National Research Council, From Research to Operations in Weather Satellites and Numerical Weather Prediction: Crossing the Valley of Death, The National Academies Press, Washington, D.C. (2000).

[29] National Research Council, Satellite Observations of the Earth's Environment: Accelerating the Transition of Research to Operations, The National Academies Press, Washington, D.C. (2003).

[30] National Research Council, Assessing Requirements for Sustained Ocean Color Research and Operations, The National Academies Press, Washington, D.C. (2011). 